\title{
Research Paper \\ Comparing the Effectiveness of Schema Therapy and Dialectical Behavior Ther- apy (DBT) on Health-Promoting Lifestyle (HPL) and Quality of Life (QoL) in HIV/ AIDS Patients
}

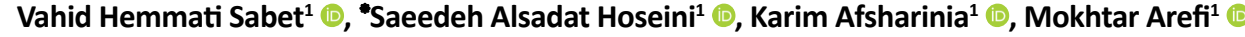

Article Info:

Received: 01 Dec 2020

Accepted: 21 Jun 2021

Available Online: 01 Oct 2021

Key words:

Schema the Bbehavior Therapy, Healthpromoting Lifestyle, Quality of Life, HIV

\section{ABSTRACT}

Background and Aim The main objective of the current study was to compare the effectiveness of schema therapy and dialectical behavior therapy (DBT) on health-promoting lifestyle (HPL) and quality of life (QoL) of HIV/AIDS patients.

Methods \& Materials The current study was an experimental study based on a pre-test-post-test design with a control group, and based on the objectives, it can be considered an applied study. The statistical population of the study included all HIV patients monitored by behavioral disorders clinics of the Healthcare Organization Centers in Hamedan County in Iran in 2019-2020. Using simple random sampling method, 45 patients were randomly selected from Shohada Clinic in Hamedan as the sample of the study, followed by randomly assigning them to three 15-member groups, i.e., two intervention groups and a control group. In order to measure the research variables, the Health-Promoting Lifestyle Profile (HPLP) (1987) and the Short Form (36) Health Survey (SF-36, 2001) were utilized. The obtained data were analyzed using ANCOVA in SPSS v. 12.

Ethical Considerations The Ethics Committee of Hamedan University of Medical Sciences approved the study (Code: IR.UMSHA.REC.1398.1063). This study has been registered in the Iranian Registry of Clinical Trials (Code: IRCT20120215009014N348).

Results The results of ANCOVA indicate that schema therapy and DBT affect HPL and quality of life, and there was a significant difference between the two groups $(P<0.001)$.

Conclusion The results show that while both therapy methods affect the selected variables, schema therapy is more effective than DBT on HPL and Quality of Life (QoL).

\section{Extended Abstract}

\section{Introduction}

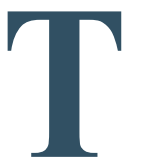

he Human Immunodeficiency Virus (HIV)/Acquired Immunodeficiency Syndrome (AIDS) is a highly challenging and overwhelming disease with numer- ous consequences [1]. One of the crucial issues related to HIV patients, which has attracted very little attention, is a lifestyle [6]. When selecting a lifestyle for improving one's health and preventing diseases, the individual engages in behaviors, such as following suitable nutrition, considering good levels of sleep and activity, maintaining a good shape, refraining from smoking and drinking alcohol, and taking protective measures against various

\section{* Corresponding Author:}

Saeedeh Alsadat Hoseini, PhD.

Address: Department of Psychology, Kermanshah Branch, Islamic Azad University, Kermanshah, Iran.

Tel: +98 (912) 8456499

E-mail: saeedeh_hosseini@iauksh.ac.ir 
diseases [8]. Health-Related Quality of Life (HRQoL) is defined as the individual's satisfaction with all the aspects of their life [13]. Schema therapy is a novel integrated treatment method, which provides a regular plan for identifying and adjusting Early Maladaptive Schemas (EMS) formed in the individual's past. The primary basis for schema therapy is classic cognitive-behavioral therapy [17]. Dialectical Behavior Therapy (DBT) emphasizes structure by considering pathological symptoms inefficient problem solving [19]. The main objective of the current study was to compare the effectiveness of schema therapy and DBT on Health-Promoting Lifestyles (HPL) and Quality of Life (QoL) in HIV/AIDS patients.

\section{Materials \& Methods}

This was an experimental study with pre-test-post-test and a control group design. Regarding the objectives, the current study is an applied research. The study's statistical population included all HIV patients monitored by behavioral disorders clinics of the Healthcare Organization of Hamedan Province of Iran in 2019-2020. Using the simple random sampling method, 45 patients were randomly selected from Shohada Clinic in Hamedan City, Iran, as the study sample, then randomly assigned them into three 15-member groups, i.e., two intervention groups and a control group. Then, 8 Schema Therapy (ST) sessions were administered for the first intervention group, and 8 DBT sessions were administered for the second intervention group. Data collection tools were: The Health-Promoting Lifestyle Profile (HPLP) was designed by Walker et al. in 1987. This questionnaire includes 52 items, measuring 6 components. These 6 aspects include nutrition, physical activity, health responsibility, stress management, interpersonal support, spiritual growth [27]. The Short Form (36) Health Survey (SF-36) includes 36 items, and 8 subscales were designed by Ware $\&$ Sherbourne 1992. The 8 subscales include physical functioning, physical role functioning, emotional role functioning, energy/fatigue, emotional wellbeing, social functioning, pain, and general health. Moreover, using two primary subscales, physical and mental health, are obtained [28].

\section{Results}

Multivariate Analysis of Variance (MANCOVA) was employed for data analysis. The collected data were analyzed in SPSS. Table 1 reports the Mean \pm SD scores of the subscales of HPL and QoL in the 3 surveyed groups. According to significant F-value calculated for comparison between mean values of two schema therapy and dialectical behavior therapy groups, Lametrix post hoc test was employed for variables, including HPL (nutri- tion, physical activity, health responsibility, stress management, interpersonal relations, \& spiritual growth) and quality of life QoL (physical health, mental health). According to Table 2, there was a significant post-test difference between experimental groups, i.e., affected by ST and DBT regarding values of HPL components; nutrition (2.722), physical activity (2.781), health responsibility (3.724), stress management (3.716), interpersonal relations (2.964), and spiritual growth (4.846) and QoL components; physical health (2.582) and mental health (2.989) ). According to the MANCOVA data (based on the odds of 0.99), the research hypothesis was confirmed; thus, ST and DBT provided a different effect on HPL, as well as the QoL in patients with $\operatorname{HIV}(\mathrm{P}=0.001,<\mathrm{P}=0.01$ respectively).

\section{Discussion \& Conclusion}

To explain the findings, in ST, by identifying EMSs through cognitive and experiential techniques and behavioral pattern-breaking, the individual's lifestyle and QoL can be improved. The maladaptive schemas of HIV patients often create problems with lifestyle and QoL; thus, modifying the schemas of these individuals can somewhat result in changes in the openness to experience. HIV patients may experience social isolation, failure, and dysfunctional schemas. These schemas can affect the individual due to effects and deficiencies that lead to a repetitive life and the inability to notice new experiences and events. Furthermore, concerning the superiority of ST, this therapeutic method focuses on cognitive, emotional, and behavioral themes; therefore, it can be helpful through techniques such as establishing relations between current problems and the schemas, evaluating the benefits and disadvantages of coping mechanisms, challenging schemas, imaginary dialogue, mental visualization, overcoming the barriers for changing behaviors, and creating significant life changes.

Moreover, the multidimensional nature of ST makes it more effective than DBT. The present study results revealed that ST, to identify underlying patterns of thought and challenge them, explicitly focusing on relation therapy, paying attention to the past and the present, attempting to break the patterns of maladaptive behaviors; applying experiential techniques, is a more comprehensive therapeutic method compared to DBT. Therefore, it was more effective in changing the HPL and QoL of individuals compared to DBT. 
Table 1. The Mean \pm SD scores of the research variables

\begin{tabular}{|c|c|c|c|}
\hline \multicolumn{2}{|c|}{ Statistical Measures } & \multicolumn{2}{|c|}{ Mean \pm SD } \\
\hline Variables & Group & Pre-test & Post-test \\
\hline & ST & $13.27 \pm 1.22$ & $20.47 \pm 2.20$ \\
\hline \multirow[t]{3}{*}{ Nutrition } & DBT & $12.33 \pm 1.29$ & $16.73 \pm 1.28$ \\
\hline & Control & $12.60 \pm 1.45$ & $12.93 \pm 1.33$ \\
\hline & ST & $14.13 \pm 7.24$ & $20.67 \pm 2.22$ \\
\hline \multirow[t]{3}{*}{ Physical activity } & DBT & $13.40 \pm 0.986$ & $16.87 \pm 0.99$ \\
\hline & Control & $13 \pm 1.60$ & $13.67 \pm 1.54$ \\
\hline & ST & $38.47 \pm 3.42$ & $50.53 \pm 1.99$ \\
\hline \multirow[t]{3}{*}{ Health responsibility } & DBT & $43.27 \pm 1.83$ & $48.13 \pm 0.99$ \\
\hline & Control & $40.67 \pm 1.95$ & $41.93 \pm 1.98$ \\
\hline & ST & $7.33 \pm 1.35$ & $16.80 \pm 2.37$ \\
\hline \multirow[t]{3}{*}{ Stress management } & DBT & $7.13 \pm 1.41$ & $12.60 \pm 1.12$ \\
\hline & Control & $8.60 \pm 1.45$ & $9.40 \pm 1.55$ \\
\hline & ST & $13 \pm 1.36$ & $19.93 \pm 1.98$ \\
\hline \multirow[t]{3}{*}{ Interpersonal relations } & DBT & $12.53 \pm 1.25$ & $16.60 \pm 1.18$ \\
\hline & Control & $12.47 \pm 1.19$ & $13 \pm 1.31$ \\
\hline & ST & $20.047 \pm 1.85$ & $28.53 \pm 2.26$ \\
\hline \multirow[t]{3}{*}{ Spiritual growth } & DBT & $20.40 \pm 2.41$ & $25.47 \pm 1.68$ \\
\hline & Control & $20.73 \pm 2.22$ & $21.73 \pm 2.22$ \\
\hline & ST & $33.33 \pm 2.32$ & $25.20 \pm 1.86$ \\
\hline \multirow[t]{3}{*}{ Physical health } & DBT & $31 \pm 1.51$ & $27.20 \pm 1.26$ \\
\hline & Control & $31.27 \pm 1.03$ & $30.33 \pm 1.11$ \\
\hline & ST & $71.07 \pm 1.71$ & $62.27 \pm 2.99$ \\
\hline \multirow[t]{2}{*}{ Mental health } & DBT & $70.20 \pm 1.47$ & $65 \pm 1.69$ \\
\hline & Control & $71.27 \pm 1.83$ & $70.27 \pm 1.83$ \\
\hline
\end{tabular}

\section{Ethical Considerations}

\section{Compliance with ethical guidelines}

The Ethics Committee of Hamedan University of Medical Sciences approved the study (Code: IR.UMSHA.REC.1398.1063). This study has been reg- istered in the Iranian Registry of Clinical Trials (Code: IRCT20120215009014N348).

\section{Funding}

This study was extracted from the $\mathrm{PhD}$. dissertation of the first author at Department of Psychology, Kermanshah Branch, Islamic Azad University, Kermanshah. 
Table 2. The MANCOVA results per HPL and QoL for the ST and DBT groups

\begin{tabular}{|c|c|c|c|c|c|c|}
\hline Source of Scattering & SS & df & MS & $\mathbf{F}$ & $\mathbf{P}$ & Mean Difference \\
\hline Nutrition & 23.842 & 1 & 23.842 & 12.433 & 0.000 & 2.722 \\
\hline Error & 47.941 & 25 & 1.918 & & & \\
\hline Physical activity & 24.891 & 1 & 24.891 & 16.159 & 0.000 & 2.781 \\
\hline Error & 38.510 & 25 & 1.540 & & & \\
\hline Health responsibility & 44.628 & 1 & 44.628 & 18.400 & 0.000 & 3.724 \\
\hline Error & 60.635 & 25 & 2.425 & & & \\
\hline Stress management & 44.430 & 1 & 44.430 & 20.527 & 0.000 & 3.716 \\
\hline Error & 54.112 & 25 & 2.164 & & & \\
\hline Interpersonal relations & 28.269 & 1 & 28.269 & 15.534 & 0.000 & 2.964 \\
\hline Error & 45.495 & 25 & 1.820 & & & \\
\hline Spiritual growth & 75.573 & 1 & 75.573 & 35.800 & 0.000 & 4.846 \\
\hline Error & 52.774 & 25 & 2.111 & & & \\
\hline Physical health & 21.457 & 1 & 21.457 & 17.538 & 0.000 & -2.582 \\
\hline Error & 30.587 & 25 & 1.223 & & & \\
\hline Mental health & 28.745 & 1 & 28.745 & 13.038 & 0.000 & -2.989 \\
\hline Error & 55.116 & 25 & 2.205 & & & \\
\hline
\end{tabular}

\section{Authors' contributions}

All authors met the standard writing criteria based on the recommendations of the International Committee of Medical Journal Publishers.

\section{Conflicts of interest}

The authors declared no conflicts of interest.

\section{Acknowledgements}

The authors would like to wholeheartedly thank Hamedan University of Medical Sciences and all the participants in the current study. 
This Page Intentionally Left Blank 


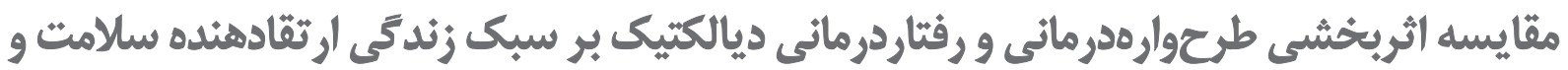

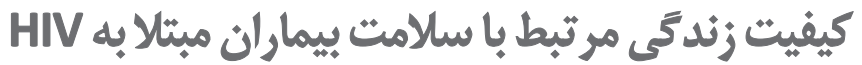

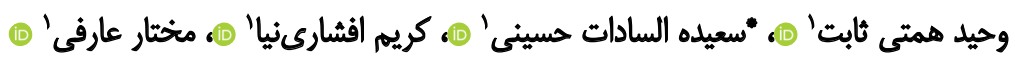

1. كروه روانشناسى، واحد كرمائشاه، دانشكاه آزاد اسلامى، كرمانشاه، ايران.

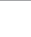

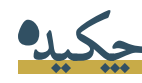

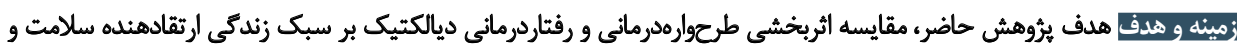

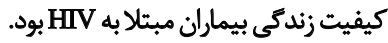

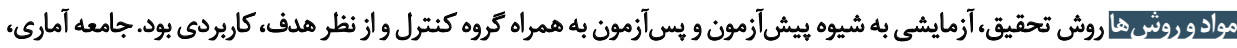

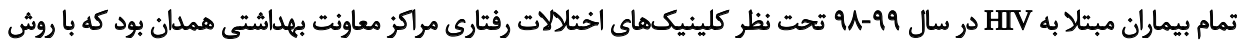

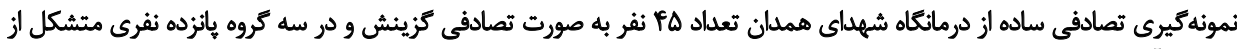

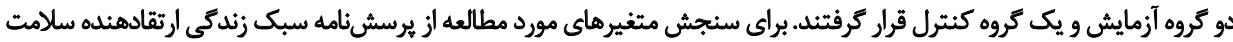

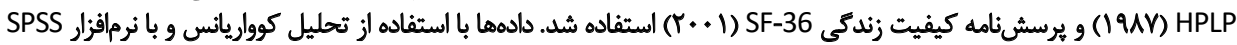
نسخه rا اتحليل شنيند.

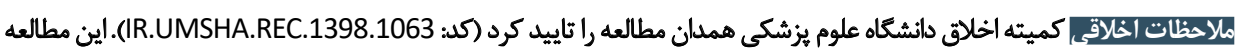
در دفتر ثبت كارآزمايى هاى بالينى ايران (كلد:

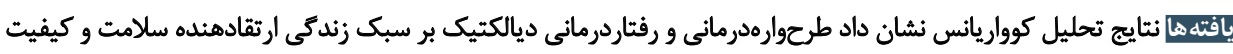

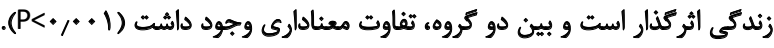

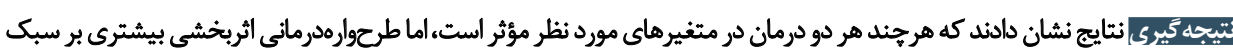

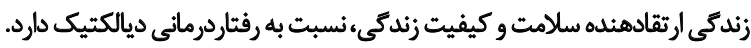

اطلاعات مقاله:

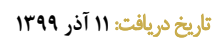

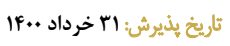

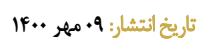

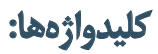

طرحوارهدرمانى، رفتاردرماني ديالكتيك،

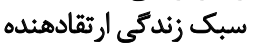

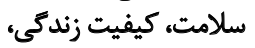
ويروس نقص ايمنى سلامن كيفي انسان

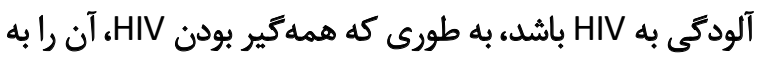

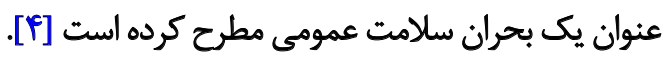

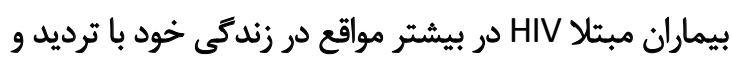

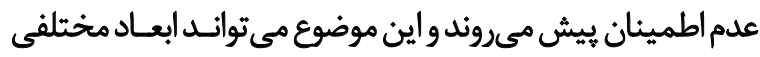

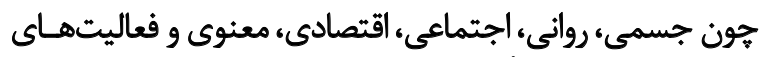
روزمره آنها را تحت تأثير قرار دهد [هـ].

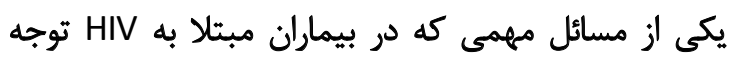

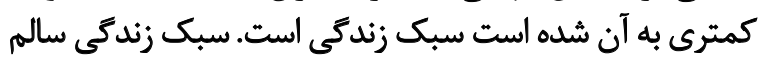

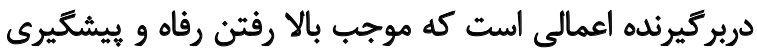

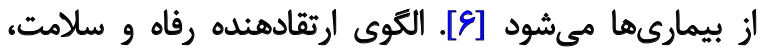

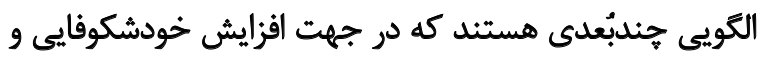

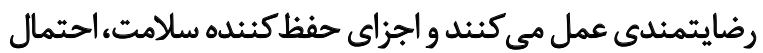

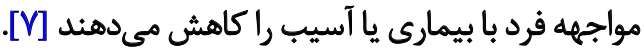

مقدمه

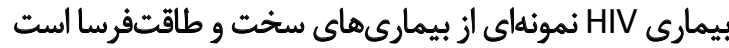

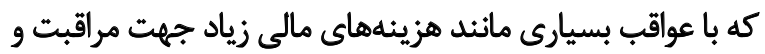

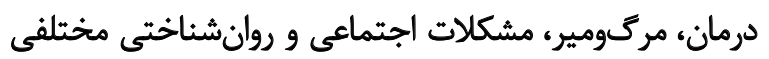

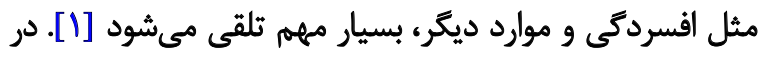

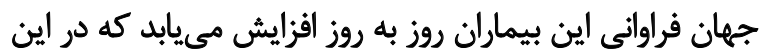

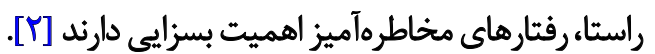

تحقيقات نشان مي دهند كه حدود 9 هو هزار بيمار مبتلابه

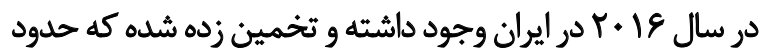

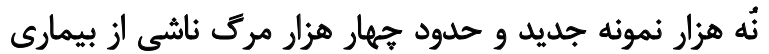

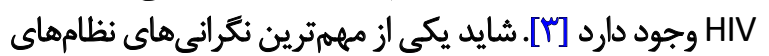

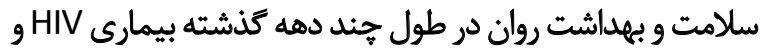

$$
\begin{aligned}
& \text { : ثويسئده مسئول: } \\
& \text { دكتر سعيده السادات حسينى مئن }
\end{aligned}
$$

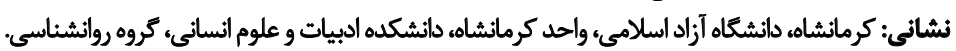

$$
\begin{aligned}
& \text { + تلفن: } \\
& \text { رايانامه: saeedeh_hosseini@iauksh.ac.ir }
\end{aligned}
$$


قابل توجهي برخوردار بوده است [19].

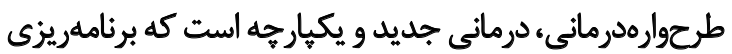

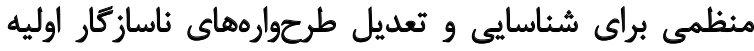

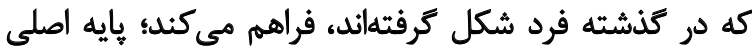

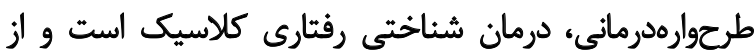

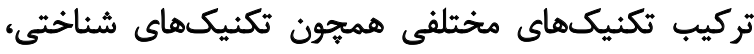

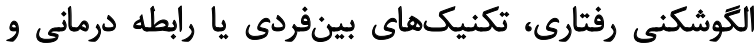

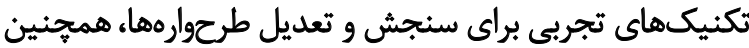

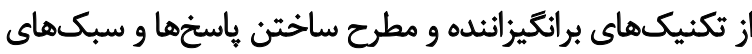

مقابلةاى استفاده مى كند [IV]

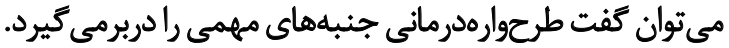

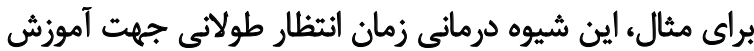

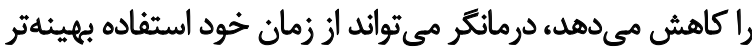

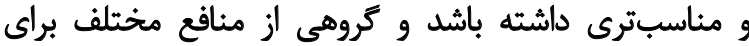

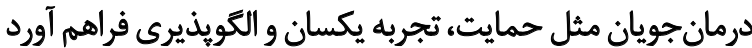

رفتاردرمانى ديالكتيك تأكيد زيادى بر ساختار دارد.اين درمان،

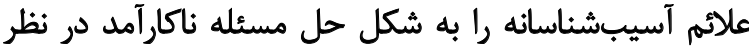

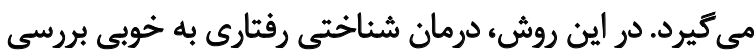

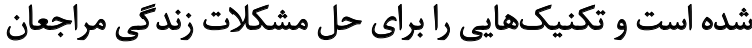

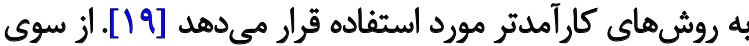

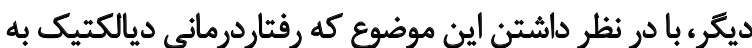

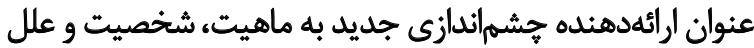

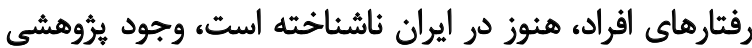

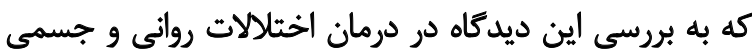

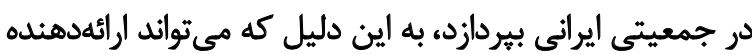

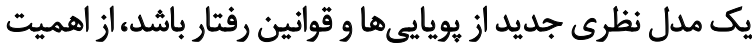

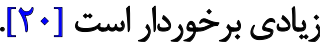

در يُؤهشى نشان داده شد، طرحوارهدرمانى و رفتاردرمانى

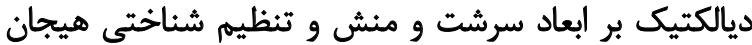

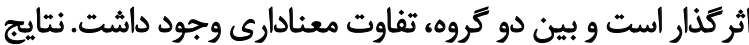

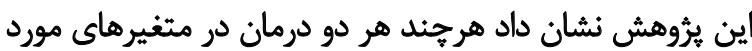

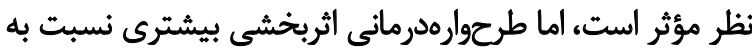

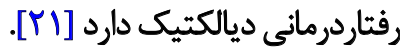

در يك يثروهش نتايج نشان داد كه طرحوار مدرمانى باعث ارتقاى

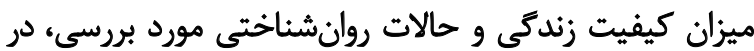

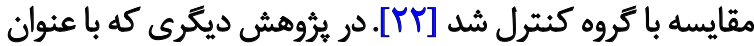

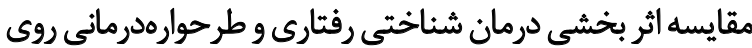

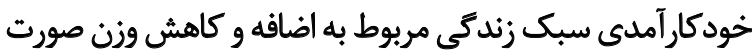

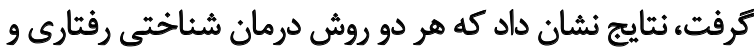

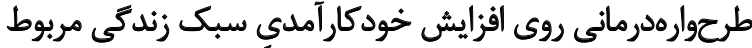

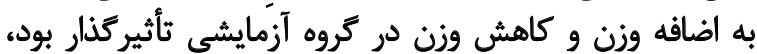

فرد با انتخاب سبك زندگى براى ارتقاى سلامت خود و

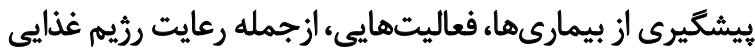

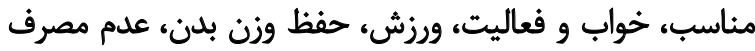

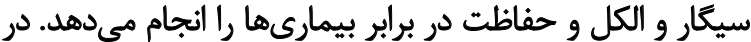

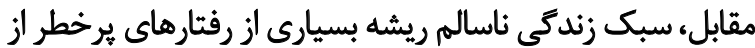

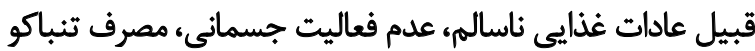

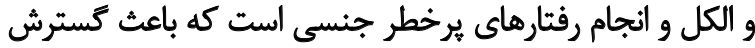

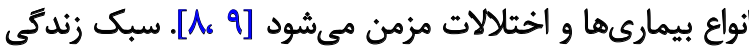

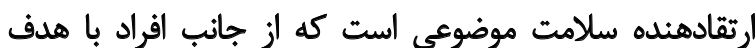

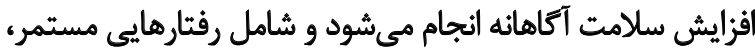

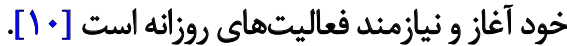

كيفيت زندكى يكى از موضوعهاى بسيار مهم در ارزيابى

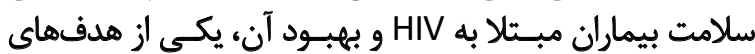

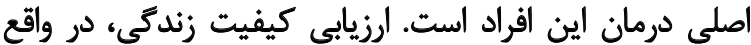

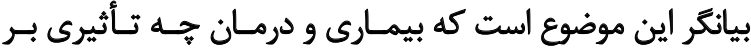

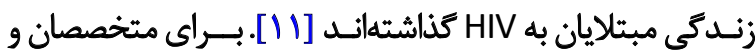

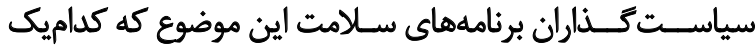

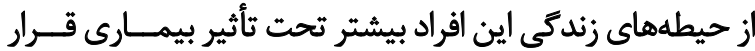

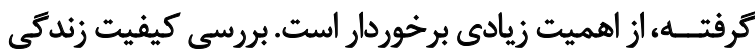

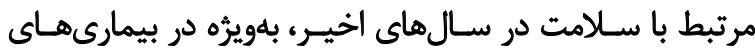

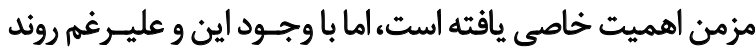

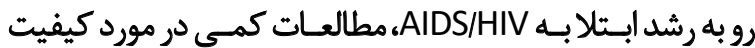

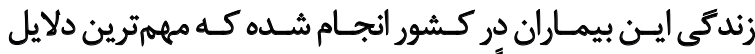

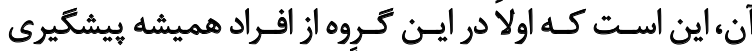

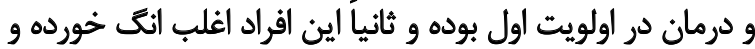

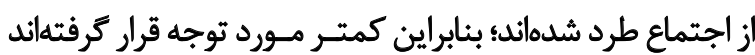

[IY]

رضايت فرداز تمام ابعاد زندكى تعريفى از كيفيت زندكى مرتبط

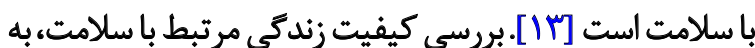

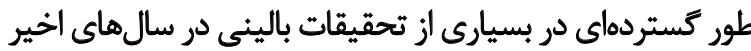

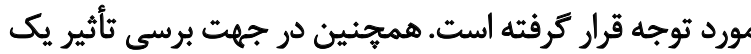

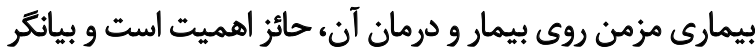

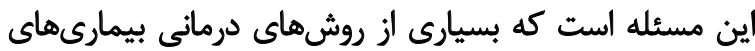

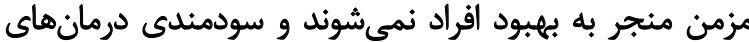

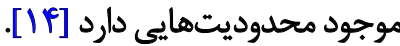

طرحوارهها، درونمايهها و الكوهاى عميق و تعميميافتهاى

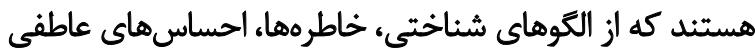

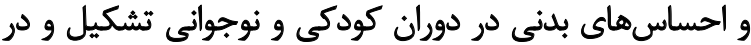

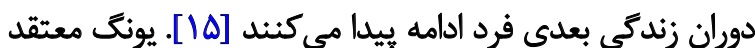

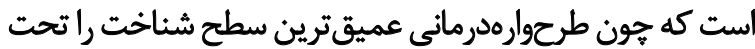

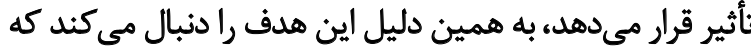

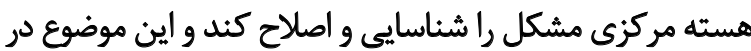

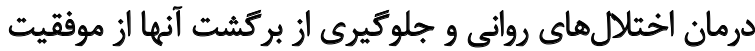


ابتلا به ساير بيمارىهاى جسمانى و روانشناختى، تحت درمان

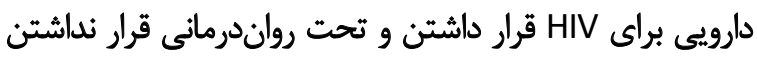

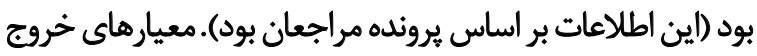

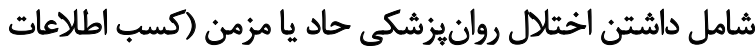

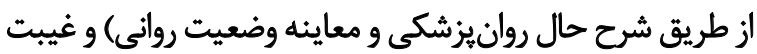
در بيش از دو جلسه درمانى بود.

ابرزارهاى سنجش يرسش وسامه سبك زندكى ارتقادهنده سلامت و وبرسش إمامه كيفيت زندكى (HPLP)

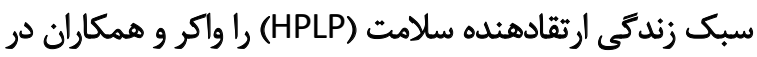

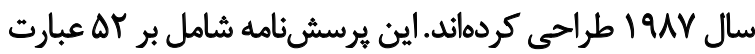

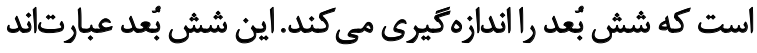

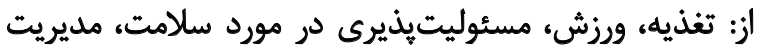
استرس (شناسايى منابع استرس و اقدامات مدئيت مديريت استرس)،

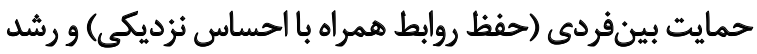

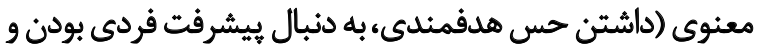
تجربه خودآكاهى و رضايتمندى).

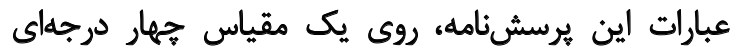

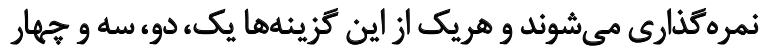

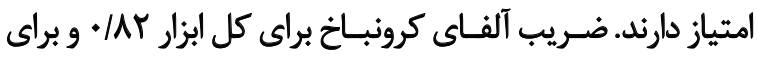

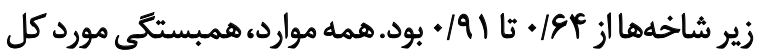

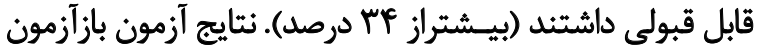

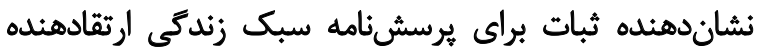

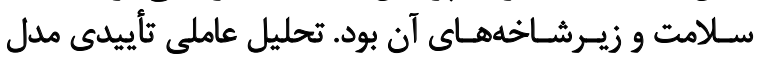

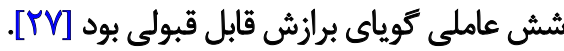

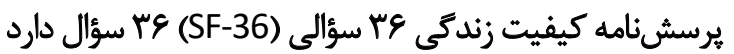

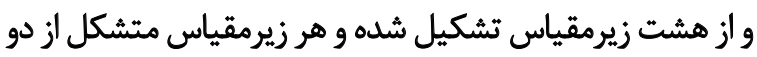

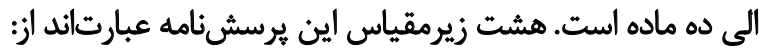

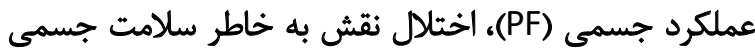

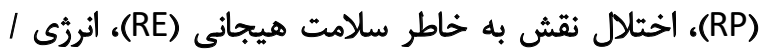

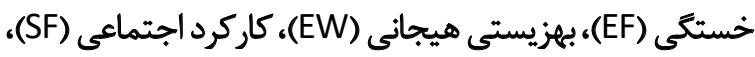
درد (P) و سلامت عمومى (GH) بيزئي (GH).

همجنين از ادغام زيرمقياسها دو زيرمقياس كلى با نامهاى

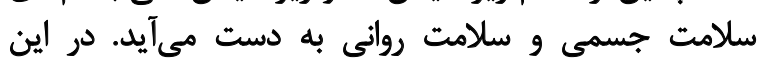

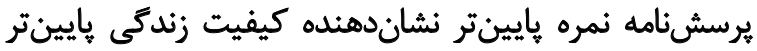

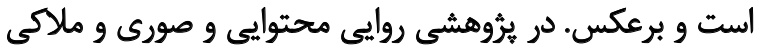

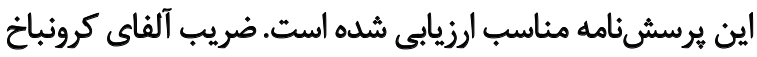

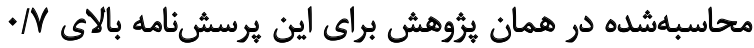

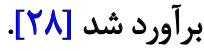

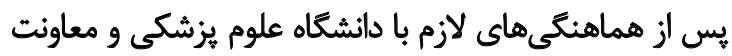

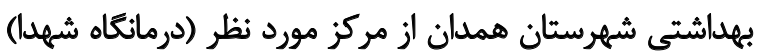

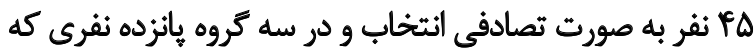

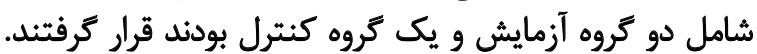

اما اثر طحوارمدرمانى نسبت به درمان شناختى رفتارى روى

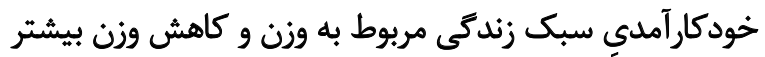

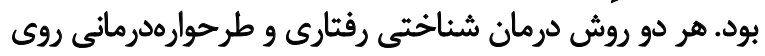

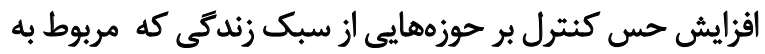

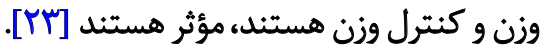

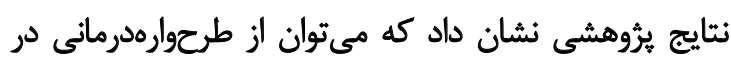

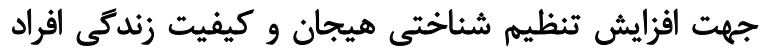

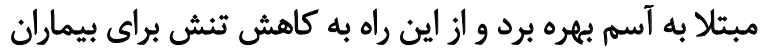

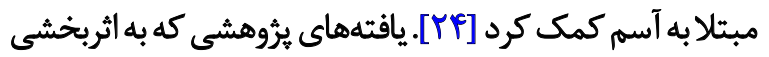

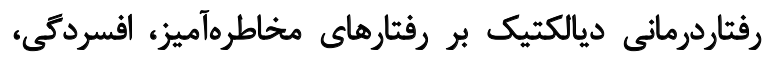

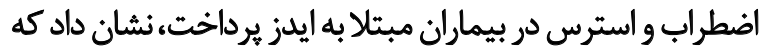

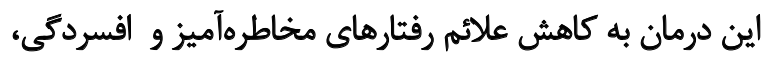

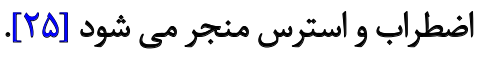

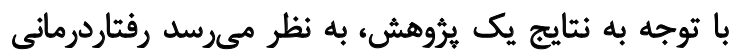

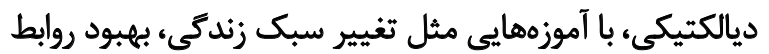

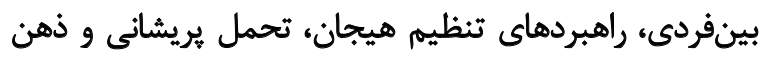

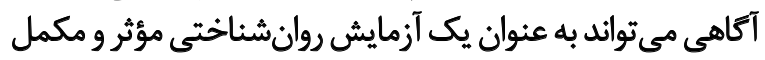

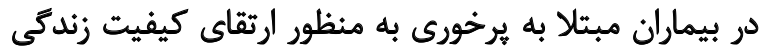
آنها، مورد استفاده قرار كيرد [عبارئ.

با عنايت به مباحث ذكرشده، هدف ئروهش حاضر مقايسه

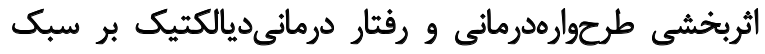

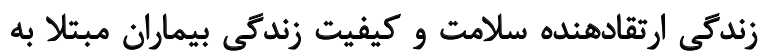

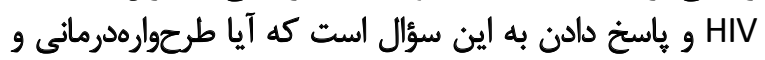

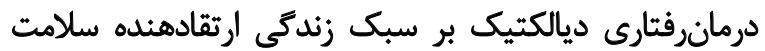

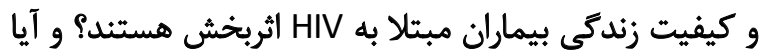

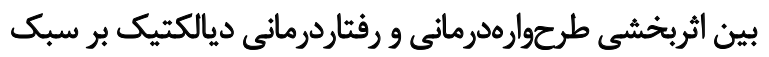

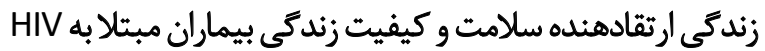

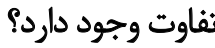

$$
\text { مواد و روشها }
$$

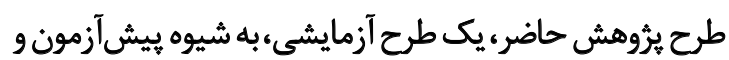

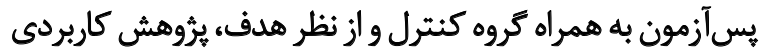

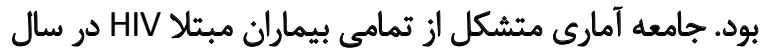

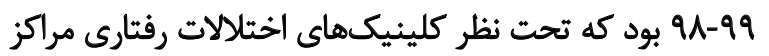

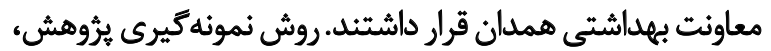

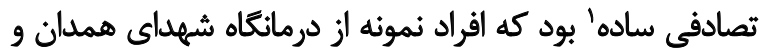

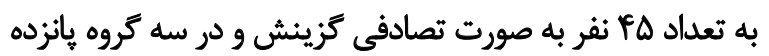

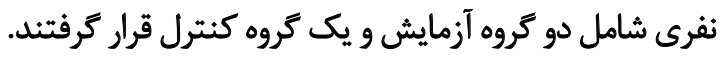
معيارهاى ورود به يثروهش شامل دامنه سنى بين بيست ثان

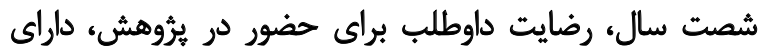

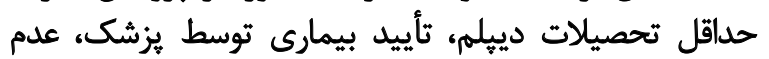

1. Simple Random Sampling 
جدول ا. خلاصه محتواى جلسات آموزشى يروتكل طرحوارددرمانى

\begin{tabular}{|c|c|}
\hline 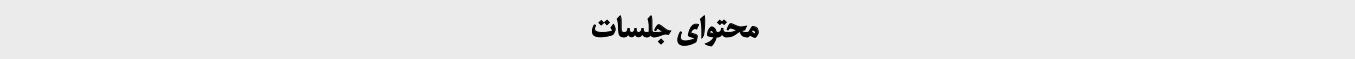 & جلسه \\
\hline 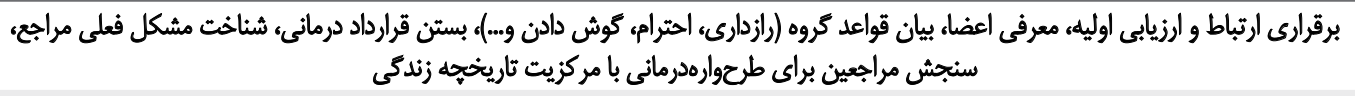 & اول \\
\hline آموزش طرحوارها و سبكهاى مقابلهاى، برقرارى ارتباط بين مشكلات فعلى وطروارهها & دوم \\
\hline (راهبردهاى شناختى)؛ ارائه منطق تكنيكهاى شناختى، بلهكاركيرى سبك درمانى روياروسازى هملانه، تعريف جديد از شواهد تأييدكتنده طرحواره & سوم \\
\hline ارزيابى كردن هزايا و معايب ياسخهاى مقابلهاي، برقرارى توفتوكو بين جنبه سالم و جنبه طرحواره، مورد هالش قرار دادن طرحوارهها، آهوزش & جهارم \\
\hline 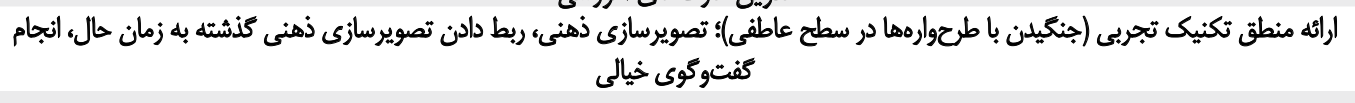 & ينجم | (1) \\
\hline 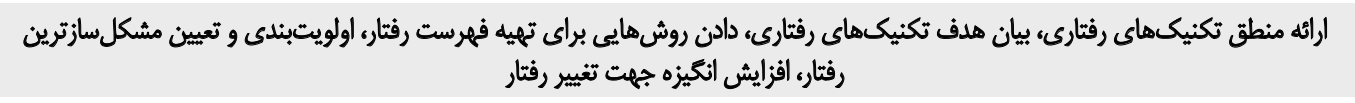 & ششم \\
\hline تكنيكهاى رفتارى، تمرين رفتارهاى سالم به وسيله تصويرسازي و ايفاى نقش، غلبه بر موانع تغيير رفتار و ايجاد كردن تغييرات برجسته در زندكى & هفتم \\
\hline مرور جهت جمعبندى جلسات كذشته. جمعبندى كردن و نتيجهيرى نهايى، تشكر از كروه و اتمام جلسات & هشتم \\
\hline
\end{tabular}

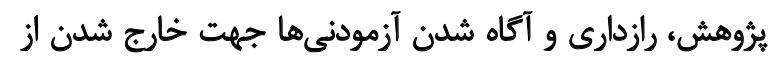

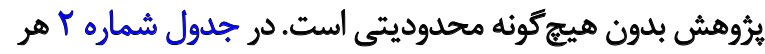

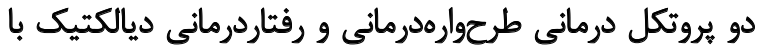
ذكر جلسات مطرح شده است.

يافتهها

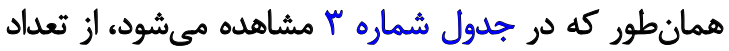

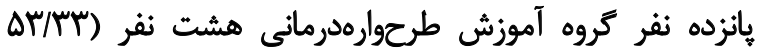

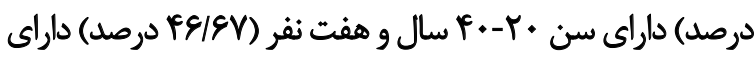

هشت جلسه طرحوارهدرمانى تروهى براى تروه آزمايش يك و

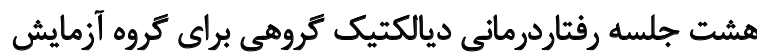
دو صورت كرفت كه مدت زمان هر جلسه نود دقيقه بود (جداني كرول

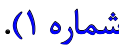

زملسات براى هر آزمايش درمانى هفتهاى دو جلسه با فاصله

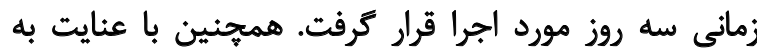

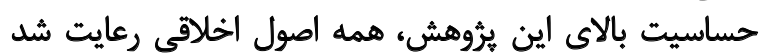

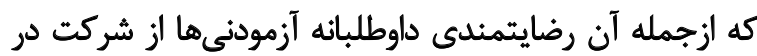

جدول r. خلاصه محتواى جلسات آموزشى يروتكل رفتاردرمانى ديالكتيك

\begin{tabular}{|c|c|}
\hline 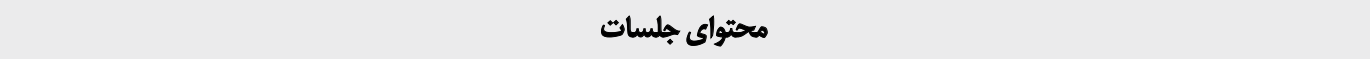 & جلسه \\
\hline 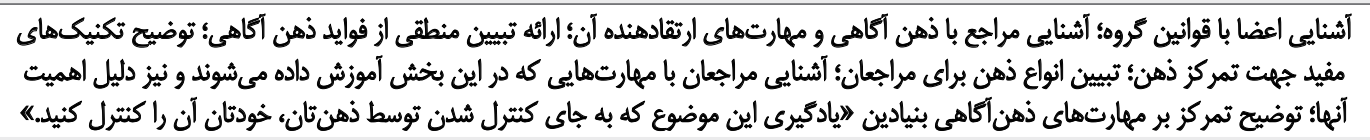 & اول \\
\hline 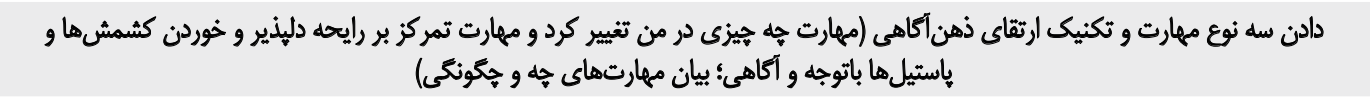 & دوم \\
\hline تبيين دلايل اهميث اثركذارى مهارتهاى بينفردى؛ أموزش اولويتها و ملزومها و اهميث ايجاد تعادل بين آنها؛ رشد تسلط و احترام به خود & سوم \\
\hline 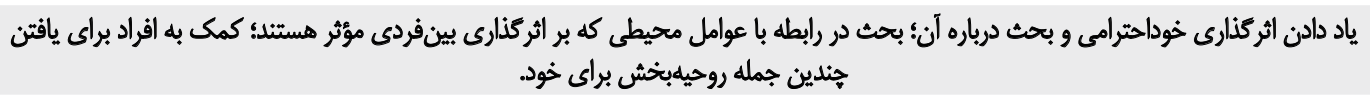 & جهارم \\
\hline 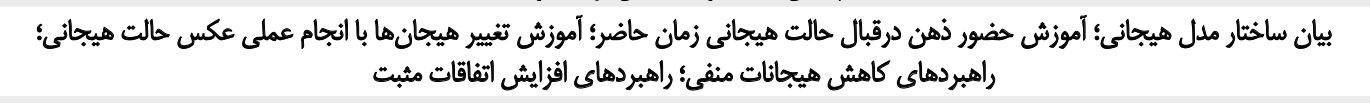 & ينجم \\
\hline أموزش حضور ذهن در برابر حالت هيجانى زمان اكنون؛ آموزش عوض كردن هيجانها با اجراى عمل متضاد حالت هيجانى & 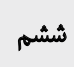 \\
\hline تبيين علت آموزش مهارتهاى تحمل آثفتكى؛ الرائه مقدماتى بحث در رابطه با مهارتهاى تحمل و بقا در شرايط بحرانى & هفتم \\
\hline أموزش راهبردهايى تحمل شامل راههاى يرت كردن حواس؛ ارزيابي نهايى مراجعان & هشتم \\
\hline
\end{tabular}


جدول ". ويرَّى هاى جمعيتشناختى گروه آموزش طرحوارهدرمانى، رفتاردرمانى ديالكتيك و گروه كنترل

\begin{tabular}{|c|c|c|c|c|}
\hline درصد فراوانى تجمعى & درصد فراوانى & ق فراوانى & سطc & مثغير \\
\hline$\Delta r / r r$ & $\Delta r / M T$ & $A$ & $r+r$. & \multirow{6}{*}{ سن } \\
\hline 1.0 & Pelav & $v$ & $f+-\varepsilon$. & \\
\hline q. & e. & 9 & r. $-f$. & \\
\hline $1 .$. & r. & 8 & $p+-8$. & \\
\hline g. & r. & $q$ & $r \cdot r$. & \\
\hline $1+$. & $\varepsilon$ & 8 & $r+q$. & \\
\hline 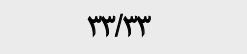 & TH/T & $\Delta$ & هجرد & \multirow{6}{*}{ تاهل } \\
\hline $1 \cdots$ & Eeler & 1. & متأهل & \\
\hline$\Delta r / T H$ & 每 & $\Delta$ & مجرد & \\
\hline $1 .$. & EqIEN & 1. & مثتهل & \\
\hline fEIAV & reler & $\checkmark$ & 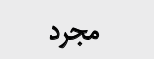 & \\
\hline $1 .$. & $\Delta r / M T$ & $\wedge$ & مثأهل & \\
\hline ar/rrw & 每 & $\wedge$ & دييلم & \multirow{6}{*}{ تحصيل } \\
\hline $1 .$. & peler & $v$ & بالاتر از دييلم & \\
\hline PeleV & peler & 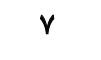 & دييلم & \\
\hline $1+\infty$ & 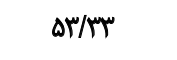 & $\Lambda$ & بالاتر از دييلم & \\
\hline geler & eqfer & 1. & دييلم & \\
\hline $1+$. & זד/Mr & $\Delta$ & بالاتر از دييلم & \\
\hline
\end{tabular}

cins

نفر (سM/سب درصد) داراى تحصيلات دييلهم به بالا بودند. همان گونه كه در جدول شماره ب ذكر شده است، آزمودنىهاى

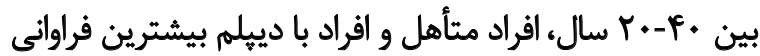

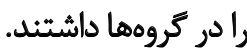

در بخش آمار توصيفى از جدول ميانكين و انحراف معيار و در

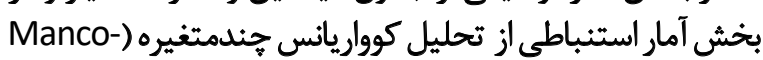
(Va

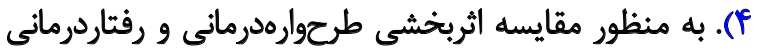
ديالكتيك بر سبك زندكى ارتقادهنده سلامت و كيفيت زند إنى

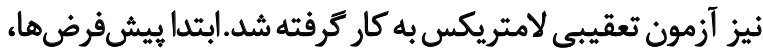

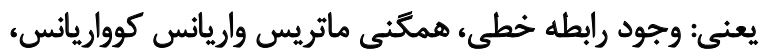

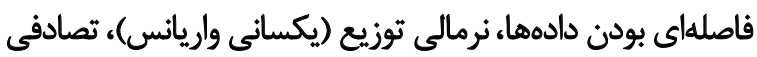

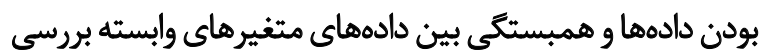

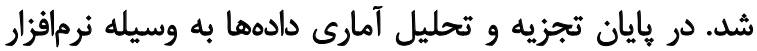

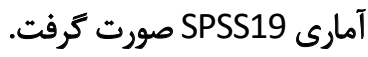

با توجه به اينكه بررسى هاى انجامگ فرته حاكى از رعايت شدن

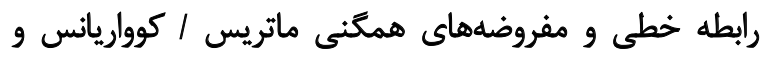

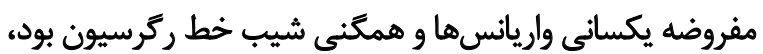

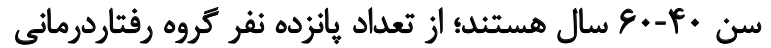

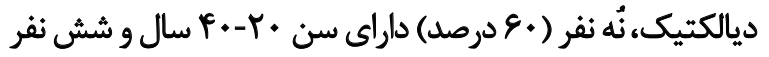

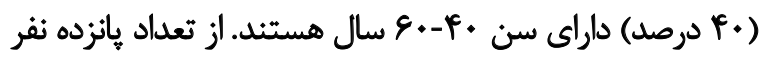

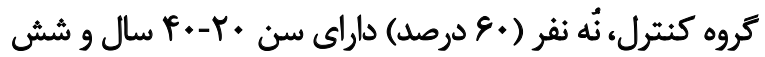

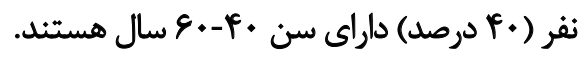
بر اساس وضعيت تأهل، از تعداد يانزده نفر كروه آموزش

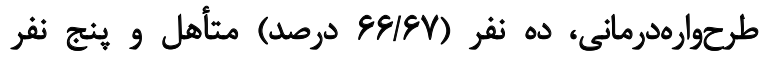

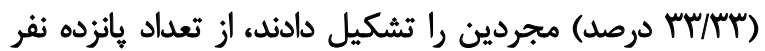

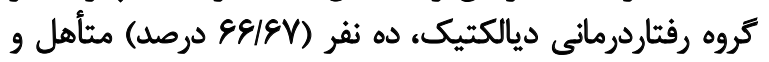

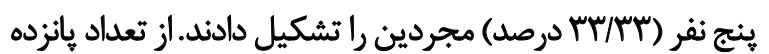

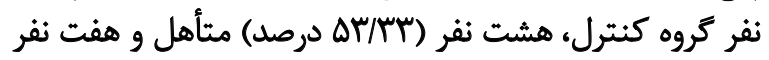

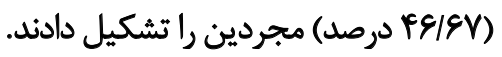

بر اساس وضعيت تحصيلات، از تعداد هانزده نفر كروه آموزش

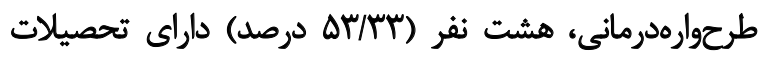

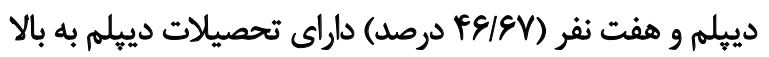

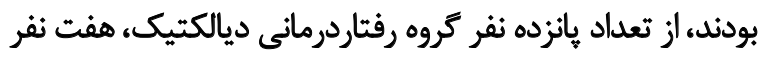

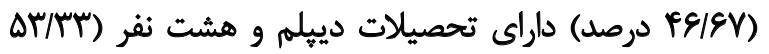

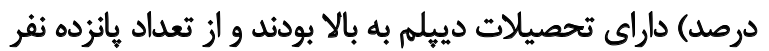

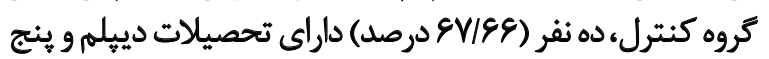


جدول F. ميانتين و انحراف معيار متغيرهاى يثروش در دو كروه آزمايش و كنترل

\begin{tabular}{|c|c|c|c|}
\hline \multicolumn{2}{|c|}{ ميانغين=|نحراف معيار } & \multicolumn{2}{|c|}{ شاخصدهاي أمارى } \\
\hline بس أزمون & يشي آزمون & تروه & مثغير \\
\hline$r+/ F \Psi T / r$. & IT/TYEV/Tr & طرحوارهدرمانى & \\
\hline $\mid \& / N^{\top} \pm 1 / K A$ & $\mid r / r r \pm 1 / T a$ & رفتاردرمانيى ديالكتيك & تغذيه \\
\hline $\mid r / a r \pm I / r r$ & $\mid r / \varepsilon+ \pm 1 / F a$ & كنترل & \\
\hline$r+/ E V \pm T / R T$ & $I F / I T \pm V / M F$ & طرحوارهدرمانى & \\
\hline $18 / A V \pm . / 99$ & $\mid w / F \cdot \pm \cdot / q \varepsilon$ & رفتاردرمانى ديالكتيك & ورزش \\
\hline $\mid r / E V \pm V / \Delta f$ & $|\Psi \pm| / \varepsilon$. & كنترل & \\
\hline$\Delta \cdot / \Delta r \pm$ I/Qq & YNFYET/NT & طرحوارهدرمانى & \\
\hline FNIH $\pm \cdot / 99$ & $r \Psi / r V \pm V / A r$ & رفتاردرمانى ديالكتيك & مسئوليتيذيرى درمورد سلامت \\
\hline$P V / q T \pm 1 / q u$ & $1 \cdot 18 V \pm 1 / 90$ & 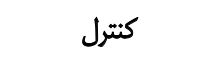 & \\
\hline $1 \& / A \cdot \pm r / M V$ & $V / \pi T \pm 1 / \Gamma \times \Delta$ & طرحوارهدرمائى & \\
\hline $\mid r / \varepsilon \cdot \pm V / T$ & V/IY士】/PI & رفتاردر مانيى ديالكتيك & مديريت استرس \\
\hline$\checkmark / \varphi+ \pm 1 / \Delta \Delta$ & $N E \cdot \pm 1 / F a$ & كنترل & \\
\hline 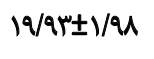 & e & طرحوارهدرمانى & \\
\hline $\mid \varepsilon / \varepsilon . \pm 1 / M$ & $\mid r / \Delta T \pm V / r \Delta$ & رفتاردرماني ديالكتيك & روابط بينفردى \\
\hline$|r \pm| r|r|$ & $1 Y / F V \pm 1 / 19$ & 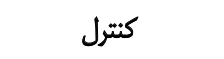 & \\
\hline RNATET/RE & $r \cdot I \cdot P V \pm I / \Lambda \Delta$ & طرحوارهدرمانى & \\
\hline$r \Delta / F Y \pm V / \& \Lambda$ & $r \cdot / T+ \pm T / F)$ & رفتاردرمانى ديالكتيك & رشد معنوى \\
\hline$T M / M T I T / M T$ & $r \cdot M T \pm T / M T$ & كتترل & \\
\hline$r \Delta / r \cdot \pm V / A C$ & $M T / M \pm T / M T$ & طرحوارهدرمانى & \\
\hline$T / T \cdot \pm V / T E$ & $|m| \pm / \Delta \mid$ & رفتثاردر مانيى ديالكتيك & سلامت جسمى \\
\hline$r \cdot / M \pm 1 / M$ & MITYEI/.r & 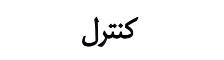 & \\
\hline ET/RYIT/Qq & $V / / \cdot v \pm 1 / M$ & طرحوارهدرمانى & \\
\hline $8 \Delta \pm 1 / 89$ & $V \cdot / r+ \pm V / F v$ & رفتاردرمانيى ديالكتيك & سلامت روائى \\
\hline$V \cdot / r V \pm V / A r$ & VITVEI/Ar & كثترل & \\
\hline
\end{tabular}

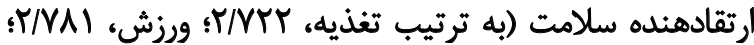

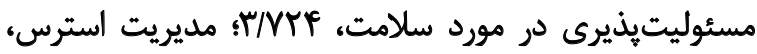

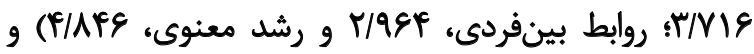

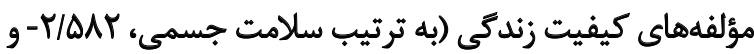

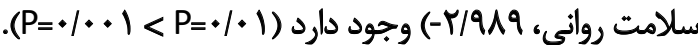

يُروهشكر مجاز است تااز تحليل كوواريانس جند متغيره استفاده

نتايج جدول شماره ه هشان مي دهد بين گروه آزمايش كه تحت

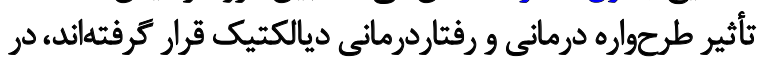

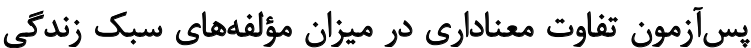


جدول ه. تحليل حاصل از تحليل كوواريانس جئدمتغيره بر اساس سبك زندكى ارتقادهنده سلامت وكيفيت زندگى گروه طرحوارهدمانى و رفتاردرمانى ديالكتيك

\begin{tabular}{|c|c|c|c|c|c|c|}
\hline تفاوت ميانغين ها & سطح معنادارى & $F$ & ميائين مجذورات & ورجه آزادى & مجموع مجذورات & هنيع يراش \\
\hline \multirow[t]{2}{*}{ T/MT } &.$\ldots$ & $\mid r / \pi T H$ & MT/AFt & 1 & Mr/AFr & تغذيه \\
\hline & & & $1 / 911$ & TA & PV/api & خطا \\
\hline \multirow[t]{2}{*}{ T/NA) } & $.1 . \ldots$ & $18 / 109$ & re/A9I & 1 & $\pi / A 91$ & مورزش \\
\hline & & & V/DF. & TA & TNAI. & خطظا \\
\hline \multirow[t]{2}{*}{$r / M T F$} & $* \ldots$ & Wr.. & Pe/gTA & 1 & RE/gTA & مسئوليت يذيرى مرد سلاهت \\
\hline & & & T/FTA & TA & $8 . / 8 \mathbb{R}^{\circ}$ & خطا \\
\hline \multirow[t]{2}{*}{$r / M V$} & $+1+\infty$ & $r+/ A T Y$ & 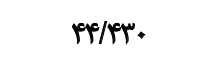 & 1 & Pe/RT. & مديريت استرس \\
\hline & & & T/Iat & ra & $\Delta F / I I T$ & خطا \\
\hline \multirow[t]{2}{*}{ r/QSF } & $+\%$ & $\mid \Delta / \Delta M$ & TNREA & 1 & rNREg & روابط بين فردى \\
\hline & & & I/AT. & ra & $P A / \% q \Delta$ & خطا \\
\hline \multirow[t]{2}{*}{ F/AFe } & .10. & $r \Delta / A .$. & $\mathrm{V} \Delta / \Delta \mathrm{VH}$ & 1 & $V \Delta / \Delta V^{\infty}$ & رشد رعنوى \\
\hline & & & $r / M I I$ & ro & $\Delta r / M P$ & خطا \\
\hline \multirow[t]{2}{*}{$-Y / \Delta A Y$} & $1 \%$ & IV/AYA & $r V / P A r$ & 1 & rI/Par & سلامت جسمى \\
\hline & & & I/TM & ro & $r \cdot / Q A Y$ & خطا \\
\hline \multirow[t]{2}{*}{$-r / u q$} & $+\cdots$ & W & ravio & 1 & TNMTQ & سلامت روانيى \\
\hline & & & $r / T \cdot \Delta$ & ro & $\Delta \Delta / 118$ & خطا \\
\hline
\end{tabular}

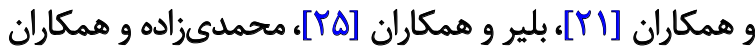

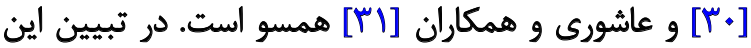

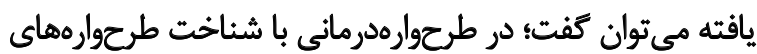

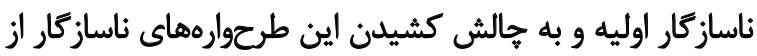

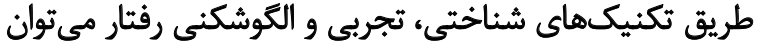

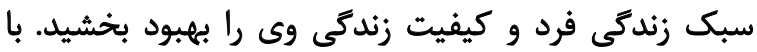

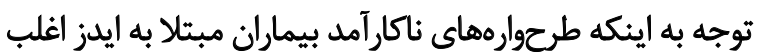

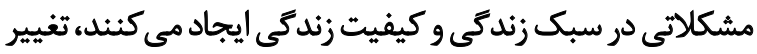

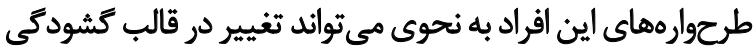

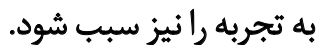

بيماران مبتلا به ايدز شايد طرحوارههاى انزواى اجتماعى،

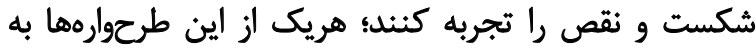

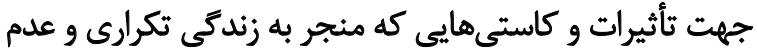

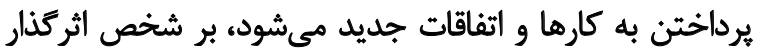

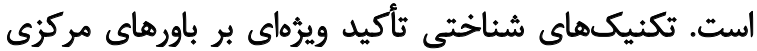

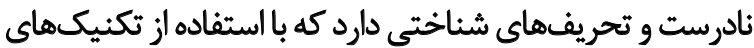

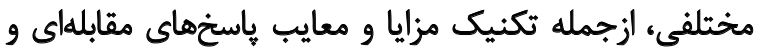

با توجه به نتايج تجزيه و تحليل اطلاعات بر اساس آزمون

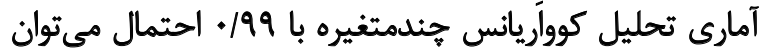

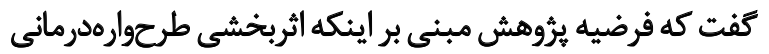

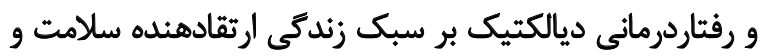

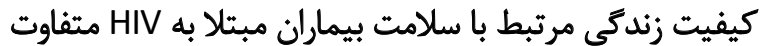

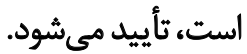

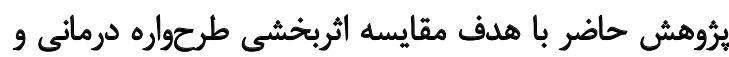

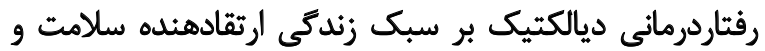

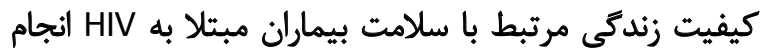

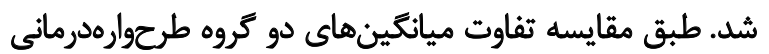

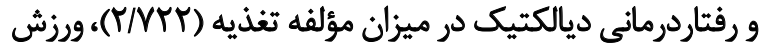

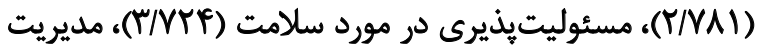

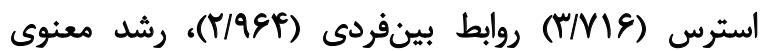

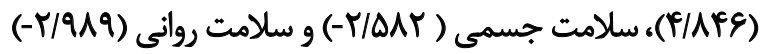

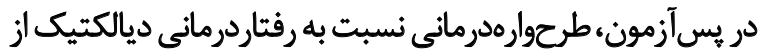

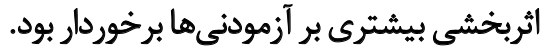

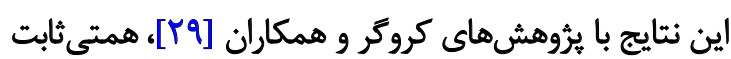


وخيمتر كند و در مسئوليتيذيرى در مورد سلامت و رشد معنوى

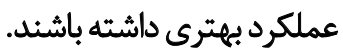

همجنين در رابطه با برترى طرحوارهدرمانى، با توجه به بهان

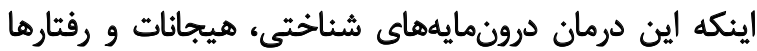

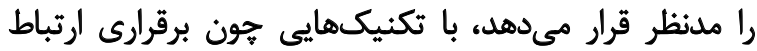

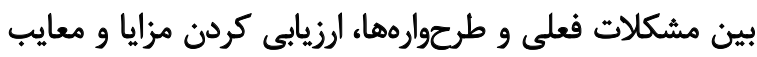

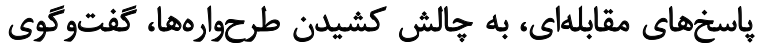

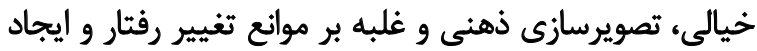

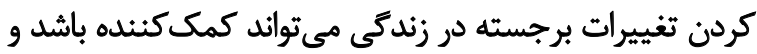

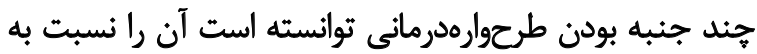
رفتاردرمانى ديالكتيك اثربخشتر كنداند

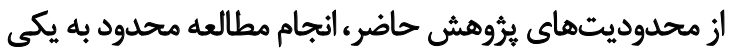

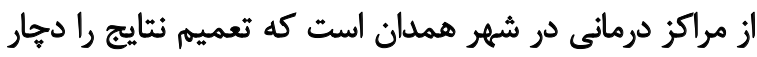

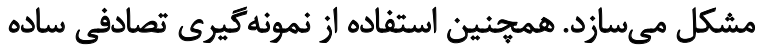

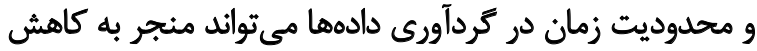

دقت نمونه كيرى و درنتيجه كاهش توان تعميم نتايج شود.

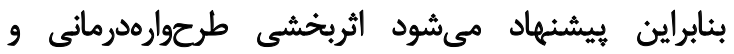

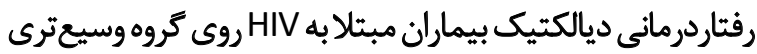

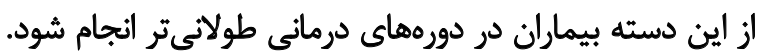

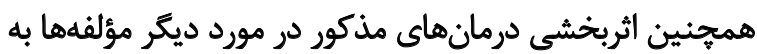
طور جداكانه و همراه با هم بررسى شودي.

نتيجهإكيرى

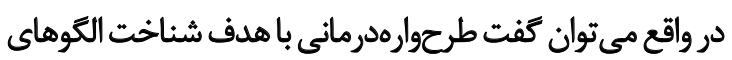

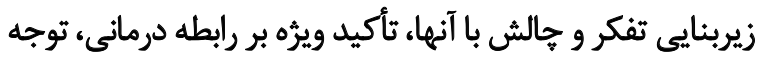

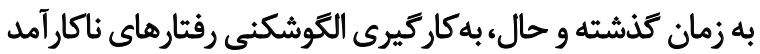

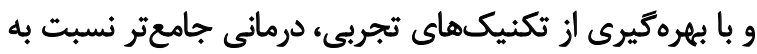

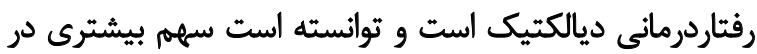

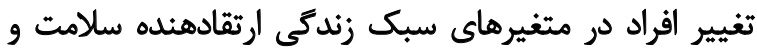

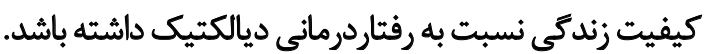

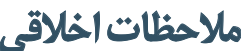

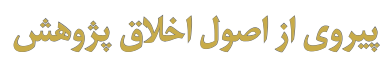

اين ثروهش در مركز ثبث كارآزمايى بالينى ايران با كد

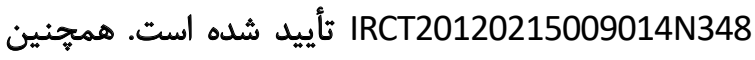
اين مطالعه داراى كد اخلاق از دانشكاه علوم يزشكى همدان به به بهن

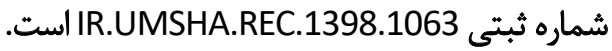

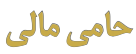

اين مقاله بركرفته از رسالهى دكترى نويسنده اول دركروه روانشناسى، دانشكده ادبيات و علوم انسانى، واحد كرئه ورمانشاه،
برقرارى كفتتوكو بين جنبه سالم و جنبه طرحواره و درنهايت

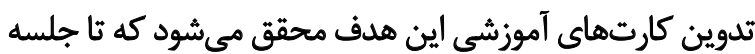

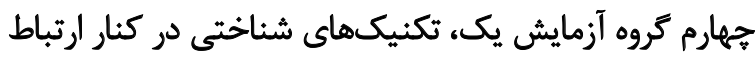
درمانى مناسب و روياروسازى همدلائه اجرا شدي.

كروه دوم، تكنيكهاى تجربى در طرحوارهد إدرمانى است

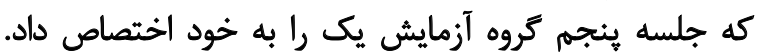

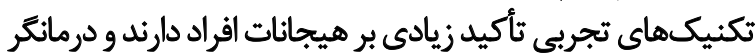

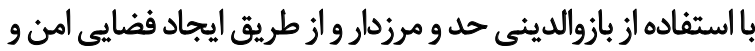

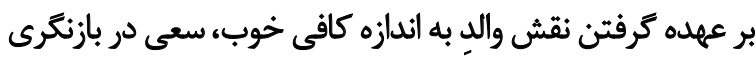

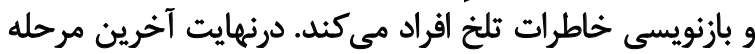

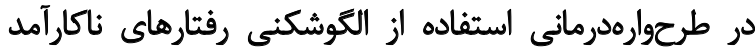

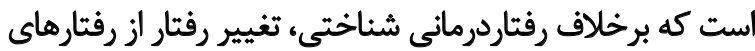

$$
\text { دردسرسازتر شروع مىشود. }
$$

در كروه آزمايش دو از جهار دسته مهارت استفاده شد. در ابتدا

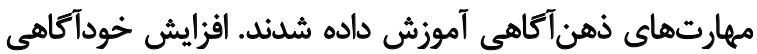

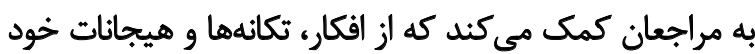

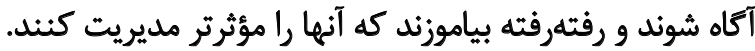

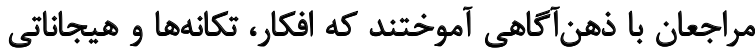

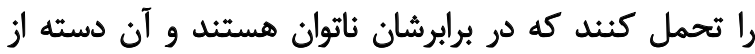

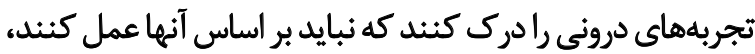

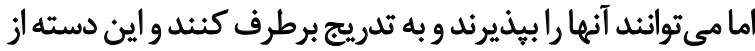

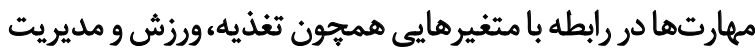
استرس به خوبى كار آمد بود.

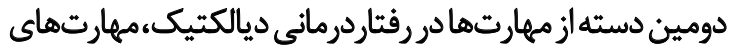

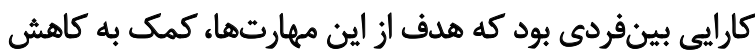

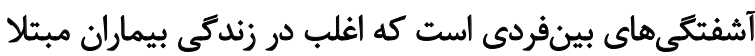

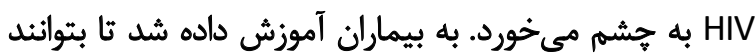

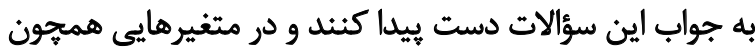

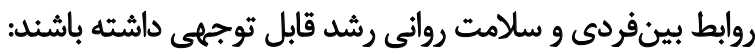

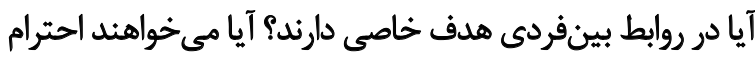

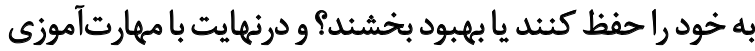
كه در اين زمينه انجام شد به اهداف خود بندئ رسيدند.

دسته سوم، مهارتهاي تنظيم هيجان است كه هدف آن

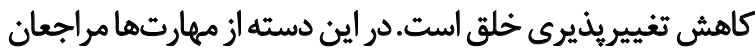

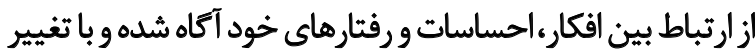

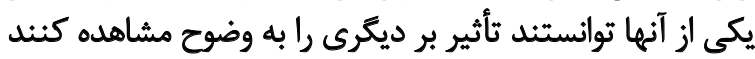

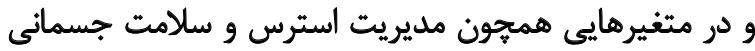

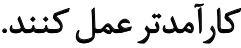

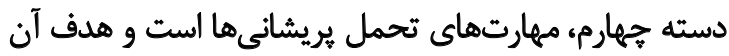

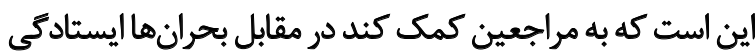

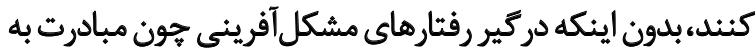

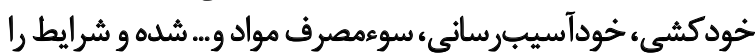


دانشًاه آزاد اسلامى، كرمانشاه است.

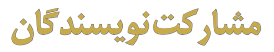

تمام نويسندكان معيارهاى استاندارد نويسندكى بر اساس

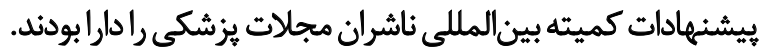

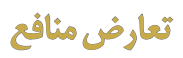

در اين مقاله براى نويسندكان تضاد منافعى وجود ندارد.

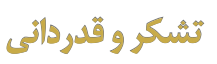

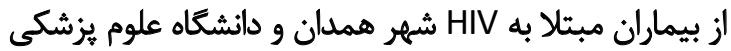

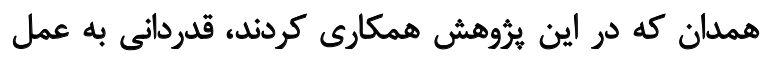

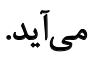




\section{References}

[1] Cane TPC. Facilitating and supporting HIV+ parenthood: Lessons for developing the advocate role of voluntary HIV support services workers. Sex Reprod Healthc. 2017; 16:186-91. [DOI:10.1016/j. srhc.2018.04.001] [PMID]

[2] King B, Winchester MS. HIV as social and ecological experience. Soc Sci Med. 2018; 208:64-71. [DOI:10.1016/j.socscimed.2018.04.015] [PMID]

[3] Global AIDS update. Geneva: UNAIDS; 2016. https://www.unaids. org/en/resources/documents/2016/Global-AIDS-update-2016

[4] Trobst KK, Herbst JH, Masters HL, Costa PT. Personality pathways to unsafe sex: Personality, condom use, and HIV risk behaviors. J Res Personal. 2002; 36(2):117-33. [DOI:10.1006/jrpe.2001.2334]

[5] Mishel MH. Theories of uncertainty in illness. In: Smith M, Liehr P, editors. Middle range theory for nursing. 3th ed. New York: Springer; 2013. http://connect.springerpub.com/content/book/978-0-82619552-4

[6] Uphold CR, Holmes W, Reid K, Findley K, Parada JP. Healthy lifestyles and health-related quality of life among men living with HIV infection. J Assoc Nurses AIDS Care. 2007; 18(6):54-66. [DOI:10.1016/j. jana.2007.03.010] [PMID]

[7] Whitehead D. Health promotion and health education: Advancing the concepts. J Adv Nurs. 2004; 47(3):311-20. [DOI:10.1111/j.13652648.2004.03095.x] [PMID]

[8] Igra V, Irwin CE. Theories of adolescent risk-taking behavior. In: DiClemente RJ, Hansen WB, Ponton LE, editors. Handbook of adolescent health risk behavior. Boston, MA: Springer; 1996. [DOI:10.1007/9781-4899-0203-0_3]

[9] Rew L, Carver T, Li CC. Early and risky sexual behavior in a sample of ruraladolescents. Issues Compr Pediatr Nurs. 2011; 34(4):189-204. [DOI:10.3109/01460862.2011.619861] [PMID]

[10] Cheng YR. Investigation and countermeasures on the health-promoting lifestyle of the disabled elderly in a rural area. Chin Nurs Res. 2015; 2(4):107-9. [DOI:10.1016/j.cnre.2015.09.007]

[11] Vahdat K, Hadavand F, Rabieian P, Farhangpour L. [Study of quality of life in patients living with HIV in Bushehr province (Persian)]. Iran South Med J. 2012; 15(2):119-26. http://ismj.bpums.ac.ir/article1-322-en.pdf

[12] Nikoo Seresht Z, Rimaz S, Asadi lari M, Nedjat S, Merghati khoie $E$, Motevallian SA, et al. [The relationship between quality of life and social capital amongst people living with HIV/AIDS attending the Imam Khomeini Hospital Consultation Center for clients with risky behaviors in Tehran- 2011 (Persian)]. J Sch Public Health Inst Public Health Res. 2014; 11(3):17-28. http://sjsph.tums.ac.ir/article1-5055-en.html

[13] Oyama $\mathrm{Y}$, Fukahori H. A literature review of factors related to hospital nurses' health-related quality of life. J Nurs Manag. 2015; 23(5):661-73. [DOI:10.1111/jonm.12194] [PMID]

[14] Abbasi A, Asaiesh H, Hosseini SA, Qorbani M, Abdollahi AA, Rouh $G$, et al. [The relationship between functional performance in patients with heart failure and quality of life (QOL) (Persian)]. Iran South Med J. 2010; 13(1):31-40. http://ismj.bpums.ac.ir/files/site1/ user files_047d40/lalerokh-A-10-3-180-decfad5.pdf

[15] Lobbestael J, van Vreeswijk M, Spinhoven P, Schouten E, Arntz A. Reliability and validity of the short Schema Mode Inventory
(SMI). Behav Cogn Psychother. 2010; 38(4):437-58. [DOI:10.1017/ S1352465810000226] [PMID]

[16] Young J, Klosko J, Weishaar M. Schema therapy: A practioner's guide. New York, NY: Guilford Press; 2003. https://books.google. com/books?id=vScjGGgJEZgC\&source=gbs_book_other_versions

[17] Videler AC, van Royen RJ, van Alphen SP. Schema therapy with older adults: Call for evidence. Int Psychogeriatr. 2012; 24(7):1186-7. [DOI:10.1017/S1041610211002638] [PMID]

[18] Skewes SA, Samson RA, Simpson SG, van Vreeswijk M. Shortterm group schema therapy for mixed personality disorders: A pilot study. Front Psychol. 2014; 5:1592. [DOI:10.3389/fpsyg.2014.01592] [PMID] [PMCID]

[19] Teimory S, Ghafariyan G, Yazdanpanah F. [The effectiveness of dialectical behavioral therapy on martial satisfaction in couples conflict (Persian)]. Res Clin Psychol Couns. 2018; 8(2):101-15. [DOI:10.22067/ IJAP.V8I2.66503

[20] Norizadeh B, Hajializadeh K. [The effectiveness of dialectical behavioral therapy on reducing anxiety and improving social functioning of patients with diabetes (Persian)]. Iran J Diabetes Metab. 2018; 17(3):130-8. http://ijdld.tums.ac.ir/article-1-5599-en.html

[21] Hemmati Sabet V, Hoseini S A, Afsharinia K, Arefi M. Comparison of the effectiveness of schema therapy and dialectical behavior therapy on temperament and character dimensions and cognitive emotion regulation strategies in patients with HIV. Iran Evol Educ Psychol J. 2021; 3(2):138-47. [DOI:10.52547/ieepj.3.2.138]

[22] Mohammad Nezhady B, Rabiei M. [The effect of schema therapy on quality of life and psychological well-being in divorced women (Persian)]. J Police Med. 2015; 4(3):179-90. [DOI:10.30505/4.3.179]

[23] Moradhaseli M, Shams Esfandabad H, Kakavand A. [A comparison of the effectiveness of schema therapy and cognitive behavioral therapy on the self-efficacy and lifestyle related to weight loss (Persian)]. J Res Psychol Health. 2016; 9(4):74-89. https://rph.khu.ac.ir/ browse.php?a_id=2619\&sid=1\&slc_lang=en\&ftxt=1

[24] Abbasi F, Moradi Manesh F, Naderi F, Bakhtiyarpour S. [The effectiveness of schema therapy on cognitive emotion regulation and quality of life in asthmatic individuals (Persian)]. Med J Mashhad Univ Med Sci. 2020; 63(2):2446-54. [DOI:10.22038/mjms.2020.16987]

[25] Belir S, Ansari Shahidi M, Mohammadi S. [Efficacy of dialectical behavior therapy on risky behaviors, depression, anxiety and stress in patients with Aids (Persian)]. Health Res J. 2018; 3(3):155-62. [DOI:10.29252/hrjbaq.3.3.155]

[26] Rahmani M, Omidi A. [The effectiveness of dialectical behavio therapy on quality of life among women with overweight and binge eating disorder: A clinical trial (Persian)]. J Rafsanjan Univ Med Sci. 2019; 18(2):133-46. http://journal.rums.ac.ir/article-1-4122-en. html

[27] Mohammadi Zeidi I, Pakpour Hajiagha A, Mohammadi Zeidi B. [Reliability and validity of Persian version of the health-promoting lifestyle profile (Persian)]. J Mazandaran Univ Med Sci. 2012; 21(1):10213. http://jmums.mazums.ac.ir/article-1-955-en.html

[28] Montazeri A, Goshtasebi A, Vahdaninia M, Gandek B. The Shor Form Health Survey (SF-36): Translation and validation study of the Iranian version. Qual Life Res. 2005; 14(3):875-82. [DOI:10.1007/ s11136-004-1014-5] [PMID]

[29] Kröger C, Harbeck S, Armbrust M, \& Kliem S. Effectiveness, response, and dropout of dialectical behavior therapy for borderline 
personality disorder in an inpatient setting. Behav Res Ther. 2013; 51(8):411-6. [DOI:10.1016/j.brat.2013.04.008] [PMID]

[30] Mohamadizadeh L, Makvandi B, Pasha R, Bakhtiar Pour S, Hafezi F. [Comparison of the efficacy of Dialectical Behavior Therapy (DBT) and Schema Therapy (ST) on impulsive behavior in patients with borderline personality disorder (Persian)]. J Guilan Univ Med Sci. 2018; 27(106):44-53. http://journal.gums.ac.ir/article-1-1663-en.html

[31] Ashouri A, Pasha R, Ahadi H, Hafezi F, Makvandi B. [Comparison the effectiveness of logo therapy and dialectical behavior therapy on general health and quality of life in patients with multiple sclerosis (Persian)]. Iran J Rehabil Res Nurs. 2018; 5(1):27-35. http://ijrn.ir/ article-1-373-en.htm 